\title{
The Tabulation of Mathieu Functions
}

\section{Introduction} Ince,

$$
d^{2} y / d x^{2}+(a-2 \theta \cos 2 x) y=0,
$$

and in which $a$ and $\theta$ are constants, arises in applied mathematics in two main groups of problems. The first group, of which Mathieu's problem of the transverse vibrations of a taut elliptic membrane is typical, consists of boundary problems relative to some partial differential equation, such as the wave equation, where the boundary is an ellipse-or possibly a hyperbola. Here a fundamental requirement is usually that $y$ shall admit the period $2 \pi$ in $x$. With a prescribed value of $\theta$, this restricts $a$ to a discrete set of characteristic numbers, each associated, in the concrete problem, with the frequency of a normal mode of vibration or some analogous quantity. In the complete solution of this class of problem, solutions of the associated equation

$$
d^{2} y / d x^{2}-(a-2 \theta \cosh 2 x) y=0,
$$

will usually also be needed.

A typical example of the second group of problems is the modulation of a radio carrier wave. Here one is concerned with either a steady state or an initial value problem for an ordinary differential equation, and the independent variable $x$ will frequently denote time. In this case both $a$ and $\theta$ are prescribed. It is known that the general solution may be written

$$
y=A e^{\mu x} \phi(x)+B e^{-\mu x} \phi(-x),
$$

where $A$ and $B$ are arbitrary constants, $\phi(x)$ is periodic in $x$ admitting the period $2 \pi$, and $\mu$ is a quantity depending on $a$ and $\theta$ termed the 'characteristic exponent'. Interest centres primarily upon whether $\mu$ is real or imaginary ${ }^{1}$ - in the former case the solution is 'unstable', whereas in the latter it is 'stable', i.e. remains bounded for all (real) values of $x$.

For the periodic solutions, $\mu=0$, so that the curves in the $a-\theta$ plane corresponding to the characteristic numbers dissect this plane into regions such that adjacent regions correspond to stable and unstable solutions respectively. For both groups of problems, then, knowledge of the characteristic numbers as functions of $\theta$ is a primary need.

\section{The periodic solutions}

The periodic solutions of (1) which admit the period $2 \pi$ fall into four classes, according as their Fourier series involve respectively cosines or

${ }^{1}$ For real values of $a$ and $\theta, \mu$ may be chosen, with no loss of generality, to be either purely real, or purely imaginary. 
sines of even or odd multiples of $x$. They are defined by Ince as follows :

$$
\begin{aligned}
c e_{2 n}(x, \theta) & =\sum_{r=0}^{\infty} A_{2 r}^{(2 n)} \cos 2 r x, \\
s e_{2 n+1}(x, \theta) & =\sum_{r=0}^{\infty} B_{2 r+1}^{(2 n+1)} \sin (2 r+1) x, \\
c e_{2 n+1}(x, \theta) & =\sum_{r=0}^{\infty} A_{2 r+1}^{(2 n+1)} \cos (2 r+1) x, \\
s e_{2 n+2}(x, \theta) & =\sum_{r=0}^{\infty} B_{2 r+2}^{(2 n+2)} \sin (2 r+2) x,
\end{aligned}
$$

corresponding to which the characteristic numbers are denoted in order by

$$
a_{2 n}, \quad b_{2 n+1}, \quad a_{2 n+1}, \quad b_{2 n+2} .
$$

In every case satisfaction of the differential equation necessitates recurrence formulae connecting three (in the first, two) successive coefficients. Elimination of the ratios of successive coefficients leads to an equation (involving a continued fraction) which expresses the relation between $a$ and $\theta$. The continued fraction forms the basis of the technique developed by Ince and Goldstein for the computation of the characteristic numbers, and this technique seems generally to have been used by later computers.

When the characteristic numbers are known, the ratios of the coefficients are calculable, but to make the coefficients definite, some normalisation rule must be chosen.

\section{Tables}

1. E. L. INCE, "Tables of the elliptic-cylinder functions," R. So. Edinb., Proc., v. 52, 1932, p. 355-423.

2. E. L. INCE, "Zeros and turning points of the elliptic-cylinder functions," ibid., 1932, p. 424-433.

1 is believed to be the only published table giving the numerical values of the functions themselves, and we therefore consider it first, before others chronologically earlier or later.

The tabular contents of no. 1 are

T. I, Characteristic numbers, p. 363. This gives $a_{0(1) 5}$ and $b_{1(1) 6}$ for $\theta=[0(1) 10(2) 20(4) 40 ; 7 \mathrm{D}]$.

T. II-XIII, p. 364-375. These give Fourier coefficients for $c e_{0(1) 5}(x, \theta)$ and $s e_{1(1) 6}(x, \theta)$, to $7 \mathrm{D}$, for the same values of $\theta$ as T. I.

T. XIV-XXV, p. 376-423. These give the functions $c e_{0(1) 5}(x, \theta)$ and $s e_{1(1) 6}(x, \theta)$ for $x=0\left(1^{\circ}\right) 90^{\circ}$ and $\theta=[1(1) 10 ; 5 \mathrm{D}]$, along with second differences.

An introduction, p. 355-362, defines the notation and the functions, outlines the methods of computation, and describes the contents of the tables. 
The normalisation rule adopted is that, if $y$ is any of the functions tabulated, then

$$
\int_{0}^{2 \pi} y^{2} d x=\pi
$$

It follows that no Fourier coefficient exceeds unity in absolute value. The sign is chosen to secure that the values of the $c e$ functions and the derivatives of the se functions shall be positive at $x=0$. As a consequence of these rules it follows that, as $\theta \rightarrow 0, c e_{0}(x, \theta) \rightarrow 1 / \sqrt{ } 2$, while for $m \geqslant 1$, $c e_{m}(x, \theta) \rightarrow \cos m x$ and $s e_{m}(x, \theta) \rightarrow \sin m x$.

Values of the functions for values of $x$ in quadrants other than the first can be obtained from the tables, since the properties of the functions as regards symmetry and antisymmetry about $x=0$ and $x= \pm \frac{1}{2} \pi$ are those of the trigonometrical functions to which they tend as $\theta \rightarrow 0$. Values for the corresponding negative values of $\theta$ can also be obtained, since changing the sign of $\theta$ in equation (1) is equivalent to replacing $x$ by $\left(\frac{1}{2} \pi-x\right)$. It is to be noted that changing the sign of $\theta$ also, in effect, interchanges $a_{m}$ and $b_{m}$ for odd (but not for even) values of $m$.

Interpolation of the functions in the $x$-direction is readily accomplished by means of the differences provided, but interpolation as regards $\theta$ would seem not to be feasible at this interval, neither in the functions nor in the characteristic numbers and Fourier coefficients.

T. 1 seems to have been completely overlooked in America; the present writer has encountered no reference to it in any American source.

The tabular contents of no. 2 are

T. I. Zeros, p. 431. This gives the zeros in degrees and decimals to $4 \mathrm{D}$, of the functions $c e_{2(1) 5}(x, \theta)$ and $s_{3(1) 6}(x, \theta)$, in the open interval $0<x<\frac{1}{2} \pi$, for $\theta=0(1) 10(2) 20(4) 40$.

T. II. Turning points, p. 432-3. This gives the turning points of the functions $c e_{1(1) 5}(x, \theta)$ and $\operatorname{se}_{2(1) 6}(x, \theta)$, for the same range of $x$ and values of $\theta$ as in Table I.

The numerical results exhibit the asymptotic approach of $c e_{m}$ and $s e_{m+1}$ as $\theta$ increases, which had been anticipated on theoretical grounds. The introduction contains formulae which give approximations to the zeros and turning points for large, and also for small, values of $\theta$.

Mention must also be made of short tables of characteristic numbers in earlier exploratory papers by Ince, not all of which are incorporated in no. 1. The papers are

3. E. L. INCE, "Researches into the characteristic numbers of the Mathieu equation," R. So. Edinb., Proc., v. 46, 1925, p. 20-29.

4.-(second paper), ibid., v. 46, 1926, p. 316-322.

5.-(third paper), ibid., v. 47, 1927, p. 294-301.

In these papers Ince used as the Mathieu equation

$$
d^{2} y / d x^{2}+(a+16 q \cos 2 x) y=0,
$$

so that the $a_{2 n+1}$ and $b_{2 n+1}$ of the later table no. 1 are interchanged. The sequence of characteristic numbers, in order of magnitude, is here 
$a_{0} a_{1} b_{1} b_{2} a_{2} a_{3} b_{3} \cdots$ as against $a_{0} b_{1} a_{1} b_{2} a_{2} b_{3} a_{3} \cdots$ in no. 1 . It is to be inferred that Ince became convinced of the desirability of the changes of parameter from $q$ to $\theta$, and of sign, during the course of the computations for no. 1 .

In no. 3 Ince develops the continued fraction technique for the computation of the characteristic numbers, exhibits it by numerical examples, and gives a table of numerical results. The table contains $a_{0} a_{1} b_{1} b_{2}$ and $a_{2}$ (in the earlier notation) for $q=[0(.1) 1 ; 5 \mathrm{D}]$. A diagram exhibiting the characteristic curves in the $q-a$ plane is also given (p. 28).

In no. 4 Ince explores more deeply an asymptotic formula for the characteristic numbers, valid when $q$ is large. In a table (p. 321) $a_{0(1) 5}$ and $b_{1(1) 6}$ (older notation) are given for $q=[0(.5) 5 ; 6 \mathrm{D}]$. Since the continued fraction process is one of successive approximation, it is a great advantage to be able to calculate directly a good first approximation, and herein lies the great value of this asymptotic development. It appears, however, that this does not discriminate between $a$ 's (or $b$ 's) adjacent in the (older) sequence, and is really an expansion for some intermediate characteristic number.

In no. $\mathbf{5}$ this last point is further explored, and the fact that the asymptotic expansion relates to the characteristic numbers of half-odd-integral order is shown by numerical instances. The tables, (I-IV, p. 296) contain $a_{0} a_{1} a_{1}, b_{1} a_{1\}} b_{2}, a_{2} a_{2\}} a_{3}$, and $b_{3} a_{3 \xi} b_{4}$, for $q=2,4.5,8,12.5,18$ (i.e. $\sqrt{ }(32 q)=k=8(4) 24)$.

Great as was Ince's published contribution to the tabulation of the Mathieu functions, it represents a considerable curtailment of what he had planned, and indeed accomplished. He had worked out, to at least 12 decimals, all the characteristic numbers which he published in no. 1 , and also, to at least 10 decimals, $a_{0(1) 4}$ and $b_{1(1) 4}$ for the values 64,100 and 144 of $\theta$. His Fourier coefficients were originally calculated (using a normalisation rule different from that ultimately adopted) to 12 decimals, and there exists a manuscript table of the twelve functions as tabulated in no. 1, to 7 decimals, with second differences. The manuscripts have been loaned to the present writer by L. J. C.

6. S. Goldstein, "Mathieu functions," Cambridge Phil. So., Trans., v. 23, 1927, p. 303-336.

What seems to be the first systematic attempt of any magnitude to give numerical results for the periodic Mathieu functions was published on unpaged folding sheets at the end of this memoir.

Goldstein writes the Mathieu equation in the form

$$
d^{2} y / d x^{2}+(4 \alpha-16 q \cos 2 x) y=0,
$$

so that he writes $4 \alpha$ where Ince writes $a$ (and $4 \alpha_{m}$ instead of $a_{m}$ and $4 \beta_{m}$ instead of $b_{m}$ ) and also uses the older parameter $q$. The tabular material consists of :

T. I-V, which give for $c e_{0}, s e_{1}, c e_{1}, s e_{2}$ and $c e_{2}$ respectively the values of $\alpha_{0}, \beta_{1}, \alpha_{1}, \beta_{2}$, and $\alpha_{2}$, to $5 \mathrm{D}$, along with the Fourier coefficients, for $q=[.1(.1) 1(.2) 4,5(5) 30,40(20) 100(50) 200 ; 5 \mathrm{D}]$. 
In the memoir Goldstein drew attention to the unsuitability of the older 'normalisation' rule whereby the coefficient of $\cos m x$ in $c e_{m}$, or of $\sin m x$ in $s e_{m}$, was taken as unity, and proposed the rule (5) above, except that he chose $2 \pi$ as the value of the integral for the function $c e_{0}$, which then tends to unity as $q$ tends to zero. Thus Goldstein's Fourier coefficients agree with Ince's, except that for $c e_{0}$ they are $\sqrt{ } 2$ times as great, while the characteristic numbers tabulated by Ince are four times those tabulated by Goldstein. Goldstein gives a very much wider range of $q(\theta)$ than Ince, or indeed any other known table. The only values which overlap with Ince's are

$\begin{array}{llrrrrr}\text { Goldstein } & q=.5 & 1 & 2 & 3 & 4 & 5 \\ \text { Ince } & \theta=4 & 8 & 16 & 24 & 32 & 40\end{array}$

Goldstein makes considerable use of the asymptotic expansion due to Ince, and in the memoir gives many more terms of it than Ince had calculated. The asymptotic expansion is further investigated in

7. S. Goldstein, 'On the asymptotic expansion of the characteristic numbers of the Mathieu equation," R. So. Edinb., Proc., v. 49, 1929, p. 210-223.

Here, after lengthy and complicated analysis, the dominant term of the difference between $a_{n}$ and $a_{n+1}$ and between $a_{n+1}$ and $b_{n+1}$ is determined. A second term is determined empirically and in T. III, p. 222, the values of $b_{1}-a_{0}, b_{2}-a_{1}, b_{3}-a_{2}$, and $b_{4}-a_{3}$ are compared with the values given by the two terms, for $q=[.5(.5) 5 ; 6 \mathrm{D}]$. T. IV, p. 222, shows that these results are good for small values of $q$ by comparing $\beta_{1}-\alpha_{0}$ and $\beta_{2}-\alpha_{1}$ with the two terms of the asymptotic expansion, for $q=[.1(.1) 1(.2) 2.6 ; 5 \mathrm{D}]$.

8. H. P. Mulholland \& S. Goldstein, "The characteristic numbers of the Mathieu equation with purely imaginary parameter," Phil. Mag., s. 7, v. 8,1929 , p. 834-840.

In this paper the authors put $q=i s$ in (7) and give the following Tables:

T. Ia, p. 839. $\alpha_{0}$ and $\alpha_{2}$ for $s=[.02(.02) .18 ; 6 \mathrm{D}]$.

T. Ib, p. 839. $\alpha_{0}, \beta_{1}, \alpha_{3}$ and $\beta_{4}$ for $s=.2(.2) 2$; and $\beta_{2}$ for $s=[.2(.2) .8 ; 6 \mathrm{D}]$.

It was found that $\alpha_{0}$ and $\alpha_{2}$, which for small $s$ are real and distinct, become equal when $s=.1836 \cdots$, and for greater values of $s$ (at least as far as $s=2$ ) are conjugate complex quantities. Similar remarks apply to $\beta_{2}$ and $\beta_{4}$. These facts explain the arrangement of, and what at first sight appear to be gaps in, the tables.

9. K. Hidaka, "Tables for computing the Mathieu functions of odd order $s e_{1}(x, \theta), c e_{1}(x, \theta), s e_{3}(x, \theta), \cdots s e_{7}(x, \theta)$, and $c e_{7}(x, \theta)$, and their derivatives," Imperial Marine Observatory, Kobe, Japan, Memoirs, v. 6, no. 2, 1936, p. $137-157$.

The author uses (essentially) Ince's notation and method of normalisation. The Tables (p. 142-157) give $b_{1(2) 7}$ and $a_{1(2) 7}$ for $\theta=[0(.1) 2.3 ; 7 \mathrm{D}]$, along with the Fourier coefficients of the functions, $B_{2 r+1}$ and $A_{2 r+1}$, and also those of the derivatives, $-(2 r+1) B_{2 r+1}$ and $(2 r+1) A_{2 r+1}$, to $7 \mathrm{D}$. 
The restriction to functions of odd order is apparently dictated by the requirements of a tidal problem for which the tables were computed. Hidaka asserts that the interpolation for $\theta$ is possible. A few tests by the present writer would indicate that while second differences may be adequate for the Fourier coefficients, the greatest possible accuracy in the characteristic numbers requires the use of fourth differences.

10. J. A. Stratton, P. M. Morse, L. J. Chu, \& R. A. Hutner, Elliptic Cylinder and Spheroidal Wave Functions, New York, Wiley, 1942, xii, 127 p.

This consists of a photographic reproduction of a paper by $\mathrm{Chu} \&$ Stratton, with the same title, published in J. Math. Phys., v. 20, 1941, p. 259-309, repaged as p. 1-51 in the book, to which have been added some numerical tables and an explanation of them, photographically reproduced from typescript. It was reviewed by H.B. in $M T A C$, p. 157-160.

As far as the elliptic cylinder functions (Mathieu functions) are concerned, the equation used is

$$
d^{2} S / d \phi^{2}+\left(b-c^{2} \cos ^{2} \phi\right) S=0,
$$

so that the relations between the parameters used here and those of Ince are

$$
\begin{array}{ll}
\theta=c^{2} / 4, & c=2 \sqrt{ } \theta, \\
a=b-c^{2} / 2, & b=a+2 \theta .
\end{array}
$$

The periodic solutions of (8) are defined by

$$
\begin{aligned}
& S e_{l}(c, \cos \phi)=\sum^{\prime} D_{n}^{l} \cos n \phi, \\
& S o_{l}(c, \cos \phi)=\sum^{\prime} F_{n}^{l} \sin n \phi,
\end{aligned}
$$

where the prime indicates summation over positive integral values of $n$ of the same parity as $l$. It will be seen that $S e$ and $S o$ correspond to $c e$ and $s e$ in Ince's notation. The normalisation rules adopted are that when $\phi=0$, $\mathrm{Se}$ and the $\phi$-derivative of $S o$ are each unity, i.e.

$$
\sum^{\prime} D_{n}^{l}=1, \quad \sum^{\prime} n F_{n}^{l}=1 .
$$

It follows that the relations between these Fourier coefficients, $D$ and $F$, and those of Ince, $A$ and $B$, are

$$
\begin{aligned}
A_{n}^{(l)} & =D_{n}^{l} / \sqrt{ } \sum^{\prime}\left(D_{n}^{l}\right)^{2}, & D_{n}^{l} & =A_{n}^{(l)} / \sum^{\prime} A_{n}^{(l)}, \\
B_{n}^{(l)} & =F_{n}^{l} / \sqrt{ } \sum^{\prime}\left(F_{n}^{l}\right)^{2}, & F_{n}^{l} & =B_{n}^{(l)} / \sum^{\prime} n B_{n}^{(l)} .
\end{aligned}
$$

The values of $b$ corresponding to the solutions $S e$ and $S o$ are denoted by $b$ and $b^{\prime}$ respectively, and the correspondence between these and the characteristic numbers of Ince will be clear from the above.

The Tables devoted to elliptic cylinder functions contain (p. 77-85) $b_{0(1) 4}$ and $b_{1(1) 4}^{\prime}[5 \mathrm{~S}]$, and the corresponding Fourier coefficients $D$ or $F$ [ 4 or $5 \mathrm{D}$ ], for $c=0(.2) 4.4$, the values $.1, .5(1) 4.5$ being intercalated. The greatest Fourier coefficient is given to $5 \mathrm{~S}$, and the others to the same number of decimals. 
11. S. Lubkin \& J. J. Stoker, "Stability of columns and strings under periodically varying forces," Quarterly Appl. Math., v. 1, 1943, p. 215-236.

Since the interest of these authors is in stability, characteristic numbers serve their purpose, and the tables at the end of the paper are restricted to these. The differential equation is taken in the form

$$
d^{2} f / d \theta^{2}+(\alpha+\beta \cos \theta) f=0,
$$

so that the relations between their parameters and those of Ince are

$$
\begin{aligned}
& \alpha=a / 4, \quad a=4 \alpha \\
& \beta=-\theta / 2(=-4 q), \quad \theta=-2 \beta .
\end{aligned}
$$

Corresponding to the sequence

$$
a_{0}, \quad b_{1}, \quad a_{1}, \quad b_{2}, \quad a_{2}, \quad b_{3}, \ldots \ldots
$$

of the characteristic numbers as used by Ince, Lubkin \& Stoker employ the notation

$$
\alpha\left(C_{0}\right), \quad \alpha\left(C_{1}\right), \quad \alpha\left(S_{1}\right), \quad \alpha\left(S_{2}\right), \quad \alpha\left(C_{2}\right), \quad \alpha\left(C_{3}\right), \quad \cdots
$$

The Tables (p. 232-235) contain

$$
\begin{aligned}
& \alpha\left(C_{0}\right) \cdots \alpha\left(S_{2}\right), \text { for } \beta=[0(.2) 4(.4) 10(1) 16(2) 20 ; 5 \mathrm{D}], \\
& \alpha\left(C_{2}\right) \cdots \alpha\left(S_{6}\right), \text { for } \beta=[0(.2) 4(.4) 6(2) 20 ; 5 \mathrm{D}] .
\end{aligned}
$$

These Tables reproduce- to 5 instead of 7 decimals, -12 out of Ince's 20 values, and for the same orders. Goldstein's results have been used for comparison, where the overlap occurs (but see 'Discrepancies', below).

It is known that there cannot co-exist, for the same values of $a$ and $\theta$, two independent solutions of (1) both admitting the period $2 \pi$. In other words, the second solution corresponding to $c e_{m}(x, \theta)$ or $s e_{m}(x, \theta)$ is not periodic. This second solution is rarely of physical importance, but is mathematically interesting - and elusive. One attempt to define and provide a basis for calculating a second solution is known.

12. S. Goldstein, "The second solution of Mathieu's differential equation," Cambridge Phil. So., Proc., v. 24, 1928, p. 223-230.

The table (p. 230) gives coefficients $b_{2 r+1}^{(2 m+1)}$ for $r=0(1) 8$ and $m=0(1) 8$ for the second solution corresponding to $c e_{1}(x, q)$ with $q=1$ [8D], the definition being

$$
i n_{1}(x, q)=2 x c e_{1}(x, q)+\sum b_{2 r+1}^{(2 m+1)} \sin (2 r+1) x .
$$

In this, the coefficient $b_{2 r+1}^{(2 m+1)}$ is the contribution to the total coefficient of $\sin (2 r+1) x$ due to $A_{2 m+1}^{(1)}$.

13. Philip M. Morse \& Pearl J. Rubenstein, Tables of Elliptic Cylinder Functions. Calculated in 1940, 3 p. of formulae and 17 p. of tables. Hectographed from typescript.

The writer is indebted to R.C.A. for a photoprint of these tables, which has enabled the following account to be given. The tables form part of 
the basis for the corresponding tables in no. 10, which have in some respects superseded them.

In connection with the periodic solutions $S e_{m}(c, \cos \phi)$ and $S o_{m}(c, \cos \phi)$, the tables give, in addition to the separation constants $b_{m}$ and $b_{m}^{\prime}{ }_{m}$, the quantities $N_{m}$ and $N^{\prime}{ }_{m}$ defined by

$$
\begin{gathered}
N_{m}=\int_{0}^{2 \pi}\left(S e_{m}\right)^{2} d \phi=\pi \sum^{\prime \prime}\left(D_{n}^{m}\right)^{2}, \\
N_{m}^{\prime}=\int_{0}^{2 \pi}\left(S o_{m}\right)^{2} d \phi=\pi \sum^{\prime \prime}\left(F_{n}^{m}\right)^{2},
\end{gathered}
$$

where the double prime indicates summation over all positive integers and zero, of the same parity as $m$, together with the factor 2 for the terms in which $n=0$.

These tables are notable, however, for the fact that they give some numerical information as regards solutions of the associated 'hyperbolic' equation. The differential equation, equivalent to (2), is taken in the form

$$
d^{2} R / d \psi^{2}+\left(c^{2} \cosh ^{2} \psi-b\right) R=0 .
$$

Clearly one solution of (16), with $b=b_{m}$, is

$$
S e_{m}(c, \cosh \psi)=\sum^{\prime} D_{n}^{m} \cosh n \psi .
$$

This solution converges slowly if $\psi$ is not small. There is, however, an alternative solution of (16) in the form of a series of Bessel functions, which has convenient asymptotic properties, and it is remarkable that the coefficients in this expansion are multiples of $D_{n}^{m}$. In fact, if

$$
R e_{m}^{1}(c, z)=\sqrt{ }(\pi / 2) \sum^{\prime} i^{m-n} D_{n}^{m} J_{n}(c z),
$$

then

$$
S e_{m}(c, \cosh \psi)=\sqrt{ }(2 \pi) \lambda_{m} R e_{m}^{1}(c, \cosh \psi) .
$$

The satisfaction of the differential equation (16) by the series in (18) with $z=\cosh \psi$-depends upon the differential equation and recurrence relations satisfied by $J_{n}(c z)$. These are shared by the functions of the second kind, $N_{n}(c z)$ in the notation of JAHNKE \& EMDE, or $Y_{n}(c z)$ in the notation of WATsON and the BAASMTC. Consequently there is an independent second solution of (16)

$$
\operatorname{Re}_{m}^{2}(c, \cosh \psi)=\sqrt{ }(\pi / 2) \sum^{\prime} i^{m-n} D_{n}^{m} N_{n}(c \cosh \psi) .
$$

With $\psi=0$ in this, $\mu_{m}$ is defined by

$$
\operatorname{Re}_{m}^{2}(c, 1)=\sqrt{ }(2 \pi) \mu_{m},
$$

and also $C_{m}$ and $\gamma_{m}$ by

$$
C_{m}^{2}=2 \pi \mu_{m}^{2}+1 / 2 \pi \lambda_{m}^{2}, \quad \cot \gamma_{m}=2 \pi \lambda_{m} \mu_{m} .
$$

Corresponding to $S o_{m}$, with the separation constant $b^{\prime}{ }_{m}$, the functions $R o_{m}^{1}$ and $R o_{m}^{2}$, and the constants $N_{m}^{\prime}, \lambda_{m}^{\prime}, \mu_{m}^{\prime}, C_{m}^{\prime}, \gamma_{m}^{\prime}$, are similarly defined. 
The first group of tables gives $b_{m}$, to $5 S, N_{m}$, to $5 S, \sqrt{ }(2 \pi) \lambda_{m}$, to $5 S$, $\sqrt{ }(2 \pi) \mu_{m}$, to $4 \mathrm{~S}, C_{m}$, to $4 \mathrm{~S}, \gamma_{m}\left({ }^{\circ}{ }^{1}\right)$, and the Fourier coefficients $D_{n}^{m}$, to $4 \mathrm{D}$, for $m=0(1) 4, c=0(.1) .2(.2) 4.4$ and 4.5 , and the corresponding values for the odd functions for $m=1(1) 3$ and the same values of $c$.

The second group of tables is easier to describe. It consists of values of $S e_{0(1) 4}(c, \cos \phi)$ and $S o_{1(1) 4}(c, \cos \phi)$ for $c=0(.5) 4.5$ and $\phi=0^{\circ}\left(5^{\circ}\right) 90^{\circ}$; [4D].

A quite casual examination has shown numerous discrepancies between these tables and those of no. 10, of which a few are not small. The reliability is therefore not high, but they contain the results of considerable numerical exploration, provide results that might be adequate for some purposes, and could be very valuable in giving first approximations to anyone who wished to achieve greater accuracy.

14. W. G. Bickley, Characteristic Numbers of the Mathieu Functions. Unpublished manuscript.

The present writer has for some years gradually compiled a table of values of the characteristic numbers for the values of $k=\sqrt{ } \theta=0$ (1) 10 . The calculations have been carried to sufficient figures to determine twelve decimals of the characteristic numbers. Results are complete as far as $a_{7}$ while some for $b_{8}$ have been obtained.

Some graphs showing the dependence of the Fourier coefficients $B_{2 r+1}^{(2 m+1)}$ upon $q$ are given in

15. I. Lotz, "Korrektur des Abwindes in Windkanälen mit kreisrunden oder elliptischen Querschnitten," Luftfahrtforschung, v. 12, 1935, p. 250-264.

Lotz follows Goldstein no. 6 and gives, in figs. 17-19, p. 259, graphs of the first four or five Fourier coefficients for $s e_{1}, s e_{3}$ and $s e_{5}$, for the range of $0<q<20$.

Although no numerical tables are given (readers being referred to no. 6 and no. 8 above) reference must be made to

16. E. JAHNKE \& F. EMDE, Funktionentafeln mit Formeln und Kurven, third ed., Berlin, 1938; or American reprint, New York, Dover Publications, 1944.

P. 283-295 give a synopsis of the formulae, following the notation of Goldstein no. 6.

Fig. 166, p. 286, gives the $\alpha-q$ curves for the ranges $-13<q<13$, $-6<\alpha<14$, for the characteristic numbers from $\alpha_{0}$ to $\beta_{7}$.

Figs. $167-177$, p. $288-293$, give various graphs of the five functions, $c e_{0}(x, q)$ to $s e_{2}(x, q)$ while figs. $178-181$, p. 294,295 , exhibit graphically the results of Mulholland and Goldstein no. 8.

Non-periodic solutions of the Mathieu equation depend on the characteristic exponent-cf. equation (3) supra. Some values associated with this exponent, and also some graphs of non-periodic solutions, are given in

17. J. G. Brainerd \& C. N. Weygandt, "Solutions of Mathieu's equation -I," Phil. Mag., s. 7, v. 30, 1940, p. 458-477. 
The Mathieu equation is used in the form

$$
d^{2} y / d t^{2}+\epsilon(1+k \cos t) y=0 .
$$

Two fundamental solutions, $g(t), h(t)$, are defined, such that $g(0)=1$, $g^{\prime}(0)=0 ; h(0)=0, h^{\prime}(0)=1$. It is shown that

$$
g(2 \pi)=h^{\prime}(2 \pi)=\cos 2 \pi \mu=b .
$$

Numerical values of $g(t)$ and $h(t)$ were determined for a range of values of $\epsilon$ and $k$ by numerical integration of the differential equation, and hence $b$ was determined. Sample graphs show $g(t), h(t)$, and their derivatives, for $\epsilon=9, k=.9$. In

T. I, p. 469, values of $b$ are given for $k=.1(.1) .6, .8(.1) 1$ and $3 \sqrt{ } \epsilon=[0(1) 6$, $8,9 ; 3 \mathrm{D}]$.

\section{Notation}

The conflicting notations used by the various writers are confusing. After considerable thought and discussion, the notation for the canonical form of the Mathieu equation to be used in a forthcoming account of the functions and their applications is

$$
d^{2} y / d z^{2}+(a-2 q \cos 2 z) y=0
$$

with Ince's notation for the characteristic numbers and for the functions, and with Ince's normalisation rule. The reason for the introduction of the coefficient 16 by Mathieu has lost its force, and the mixture of Greek and Roman letters in (1) cannot easily be defended. Physically, the square root of the numerical value of $q$ is significant, and this is also useful in defining solutions of the associated hyperbolic equations. It is proposed to use $k$ such that $k^{2}=|q|$.

Further details, and definitions of a set of functions satisfying the Mathieu and its associated equations, will be given elsewhere.

\section{Discrepancies}

As far as the writer is aware, no error has so far been reported in no. 1 or no. 2. One error in no. 6 has been reported, by Goldstein himself (in no. 7). The value of $\alpha_{1}$ for $q=2$, should be 2.33382 and not 2.33817 .

In the overlapping cases $(c=2,4, \theta=1,4)$ the separation constants given by no. 10 are in agreement with the characteristic numbers of Ince. The labour of comparing the Fourier coefficients (differently normalised) has not been undertaken.

The overlapping values in no. 11 have been compared with those of no. 6 and no. 7 . As regards no. 6 the following discrepancies occur:

Goldstein no. 6

$$
\begin{array}{ll}
q=0.3 & \alpha_{0}=-0.50534 \\
q=0.4 & \alpha_{0}=-0.77897 \\
q=0.2 & \alpha_{1}=0.55406
\end{array}
$$

Lubkin \& Stoker no. 11

$$
\begin{array}{ll}
\beta=1.2 & \alpha\left(C_{0}\right)=-0.50535 \\
\beta=1.6 & \alpha\left(C_{0}\right)=-0.77898 \\
\beta=0.8 & \alpha\left(S_{1}\right)=0.55906 .
\end{array}
$$

Since Lubkin \& Stoker had compared their results with Goldstein's, one may presume that they have checked the end figures of the first two of these. Differences indicate that in the third of the above, Goldstein is correct. 
Comparison of no. 1 with no. 11 yields the following:

Ince no. 1

\begin{tabular}{|c|c|c|}
\hline 40 & $u_{2}=$ & \\
\hline$=2$ & $b_{3}=$ & \\
\hline$=4$ & & 0271 \\
\hline$=24$ & $b_{4}=$ & 13 \\
\hline-12 & & 22.9 \\
\hline & $b_{6}=$ & 26. \\
\hline & $b_{6}$ & 22 \\
\hline & & 41. \\
\hline & & \\
\hline
\end{tabular}

Lubkin E Stoker no. 11

$$
\begin{aligned}
& \beta=20 \alpha\left(C_{2}\right)=-5.05198(=20.20792 \div 4) \\
& \beta=1 \alpha\left(C_{3}\right)=2.28515(=9.14060 \div 4) \\
& \beta=2 \alpha\left(S_{3}\right)=2.66777(=10.67108 \div 4) \\
& \beta=12 \alpha\left(S_{4}\right)=3.38817(=13.55268 \div 4) \\
& \beta=6 \quad \alpha\left(C_{4}\right)=5.74803 \text { (error) } \\
& \beta=16 \quad \alpha\left(C_{5}\right)=6.52721(=26.10884 \div 4) \\
& \beta=20 \alpha\left(C_{5}\right)=5.58302(=22.33208 \div 4) \\
& \beta=20 \alpha\left(S_{5}\right)=10.33749(=41.34996 \div 4) \\
& \beta=20 \alpha\left(S_{6}\right)=10.35813(=41.43252 \div 4)
\end{aligned}
$$

In the above, differences indicate that Lubkin \& Stoker's value of $\alpha\left(C_{4}\right)$ for $\beta=6$ is in error, and should be 5.74303. In the other cases the discrepancies are too small for the available differences to discriminate with certainty, but in view of the fact that all Ince's values were worked out to 12 decimals by a process of successive approximation, and bear evidence of careful checking, they are prima facie the more reliable.

Comparison of Hidaka no. 9 with Ince no. 1 shows two end-figure discrepancies

$$
\begin{array}{llr}
\text { Ince no. } 1 & \text { Hidaka no. } 9 \\
\theta=2, & B_{3}^{(5)}=0.1241361 & 0.1241360 \\
\theta=1, & a_{5}=25.0208543 & 25.0208544 .
\end{array}
$$

In the case of the second of these, the present writer agrees with Ince, having obtained 25.02085434545 ...

Comparison of Hidaka no. 9 with Goldstein no. 6 shows one discrepancy.

$$
\begin{array}{cc}
\text { Goldstein no. } 6 & \begin{array}{c}
\text { Hidaka no. } 9 \\
q=0.2-B_{3}^{(1)}=0.16171
\end{array} \quad \theta=1.6-B_{3}^{(1)}=0.1617180
\end{array}
$$

Comparison of Hidaka no. 9 with Lubkin \& Stoker no. 11 confirms that $\alpha\left(S_{1}\right)$ for $\beta=0.8$ should be 0.55406 . It yields also the following.

Hidaka no. 9

$$
\begin{array}{ll}
\theta=1.2 & b_{3}=9.0648547 \\
\theta=2.0 & b_{3}=9.1406277
\end{array}
$$

Lubkin \& Stoker no. 11

$$
\begin{array}{ll}
\beta=0.6 & \alpha\left(C_{3}\right)=2.26622(=9.06488 \div 4) \\
\beta=1.0 & \alpha\left(C_{3}\right)=2.28515(=9.14060 \div 4)
\end{array}
$$

\section{Additional References}

For collected accounts of ranges of the theory of solutions of the Mathieu equation, reference may be made (in addition to nos. 6, 10, and 16 above) to

M. J. O. STRutT, Lamésche, Mathieusche, und verwandte Funktionen in Physik undTechnik, (Ergebnisse der Mathematik, v. 1, part 3), Berlin, 1932.

E. T. Whittaker and G. N. Watson, Modern Analysis, Cambridge, University Press, fourth ed. 1927; Amer. reprint, 1943.

E. G. C. Poole, Introduction to the Theory of Linear Differential Equations, Oxford, University Press, 1936.

Imperial College of Science and Technology,

W. G. BICKLEY

LONDON, S. W. 7, England.

\section{RECENT MATHEMATICAL TABLES}

195[A, D].-J. G. Beckerley, "The calculation of arg $\Gamma(i a+1)$," Indian J. Physics, v. 15, and Indian Assoc. for the Cultivation of Science, Proc., v. 24,1941, p. $229-232.16 .5 \times 23 \mathrm{~cm}$.

$\Gamma(z)$ for complex values of $z$ has been tabulated to a rather limited extent. The tables of Walter Meissner, 1939, were reviewed in MTAC, p. 177; in his Tables of the Higher 